\title{
Viscosity Behavior of Xanthan Solutions Measured as a Function of Shear Rate
}

\author{
Katsuyoshi NISHINARI", Yutaka TANAKA ${ }^{* *}$, and Hisamoto FURUSE ${ }^{* * *}$ \\ * Department of Food and Human Health Sciences, Graduate School of Human Life Science, Osaka City University, \\ 3-3-138, Sugimoto, Sumiyoshi-ku, Osaka 558-8585, Japan \\ *** Department of Materials Science Engineering, Faculty of Engineering, University of Fukui, \\ Bunkyo, Fukui-shi, 910-8507, Japan \\ ${ }^{* * * *}$ Institute of Molecular and Cellular Biosciences, The University of Tokyo, \\ 1-1-1, Yayoi, Bunkyo-ku, Tokyo 113-0032, Japan \\ (Received : October 24, 2014)
}

\begin{abstract}
Aqueous solutions of xanthan, which is produced by Xanthomonas campestris, are used as viscosity control agents for various food materials. The viscosity is dependent on the environmental conditions affecting the xanthan macromolecule. Moreover, the viscosity frequently depends on the methods used to measure the shear rate during operation. For practical applications of xanthan solutions, the background of this phenomenon should be made clear. The solutions used in this study were prepared by dissolving xanthan in water or in an aqueous salt solution $(\mathrm{NaCl}, 1$ M). The viscosity was then measured using a rotational viscometer. For the aqueous solution of xanthan, the viscosities measured at increasing shear rates showed shear thickening in the low-shear-rate region. On the other hand, no discrepancy between the behaviors was observed when xanthan was dissolved in the salt solution. This paper discusses the aforementioned viscosity behavior using a dispersion model of polymers with contact sites for the solutions.

Key Words: Viscosity behavior / Xanthan solution / Shear rate / Solution structure / Salt ( $\mathrm{NaCl})$ solution
\end{abstract}

\section{INTRODUCTION}

The xanthan polymer, an anionic polysaccharide, is derived from the plant pathogen- Xanthomonas campestris, and is commercially obtained by cultivating the bacterium. Its polymeric structure comprises a linear chain of 1,4-linked $\beta$-D-glucopyranosyl residues with charged trisaccharide side chains of pyruvate and acetate. These side chains are attached to the corresponding residues of the polymer framework to give a pentasaccharide repeating unit. The conditions used to grow the bacterium alter the proportions of the pyruvate and acetate substituents as well as the proportions in which the xanthan polyanion associates with the mixed $\mathrm{Na}^{+}, \mathrm{K}^{+}$, and $\mathrm{Ca}^{2+}$ cations in the growth medium. ${ }^{1-5)}$

Xanthan has sufficiently high viscosity, which has a relatively low sensitivity to the salt concentration and temperature for time dependence. ${ }^{6,7)}$ Therefore, xanthan is used as a viscosity control agent in various aqueous solutions. Quantitative information about the viscosity and viscoelasticity of this

\footnotetext{
To whom corresponding should be addressed.

E-mail: hi-to.furuse@nifty.ne.jp
}

loose and sticky polymer solution is useful for its practical manipulation, and therefore, considerable effort has been devoted to studying the rheological properties of xanthan solutions. $^{8-11)}$

The physical properties of xanthan solutions, including viscosity and viscoelasticity, vary depending on the experimental conditions, i.e., salt concentration and temperature, because of the complicated structure of xanthan. ${ }^{1-23)}$ However, the fundamental shear thinning nature of viscosity holds for all the xanthan solutions, except that the viscosity behavior is different at increasing and decreasing shear rates. The present study, therefore, aims to clarify the main cause for a particular viscosity behavior.

While the viscosity of polymer solutions generally shows a monotonous decrease with the shear rate, a hysteresis behavior in the measurement of the viscosity of xanthan solutions has been observed. ${ }^{11,22)}$ That is, when the shear rate was increased in the low-shear-rate region, the viscosity increased to a maximum value and then decreased, showing shear thinning behavior. However, the viscosity showed no specific behavior for decreasing shear rates, when the sample experienced a high shear-rate. In the case of some biological 
polymer solutions, similar viscosity behaviors have been observed for increasing and decreasing shear rates. ${ }^{24,25)}$ In the study reported here, we investigated the characteristics of the solutions of xanthan dissolved in water and in an aqueous salt solution to determine whether or not there is a difference in the viscosity behavior of xanthan as a function of shear rate.

The samples used in this study were prepared by dissolving refined xanthan (Tokyo Chemical Industry, Tokyo, Japan) in water and in a salt solution $(\mathrm{NaCl}, 1 \mathrm{M})$. The viscosity of the samples was measured using a rotational viscometer to determine their shear-rate dependence. The viscosity of the xanthan dissolved in water varied with the shear rate, especially at low shear-rates, and this variation is thought to be caused by structural changes in the solution due to the shear stress generated by the changes in the shear rate. Moreover, the viscosities of the xanthan sample dissolved in the aqueous $\mathrm{NaCl}$ solution were considerably lower than those of the samples dissolved in water, and the viscosity variation at low shear-rates was relatively small. This difference might be attributed to the fact that the contacting force between the molecules weakened in the presence of $\mathrm{Na}^{+}$ions.

\section{EXPERIMENTAL}

\subsection{Sample Preparation}

The reagent used in this study was refined xanthan (Lot. No. V3HVA, TKC) derived from the bacterium $X$. campestris. Samples of $1 \%$ xanthan solutions (weight-mass/volume) were prepared by dissolving xanthan in water and in an aqueous $\mathrm{NaCl}$ (1 M) solution. Because the xanthan powder was not readily soluble in water, it was first dispersed in ethanol (99\%), which was subsequently vaporized by heating the samples in a water bath at approximately $50{ }^{\circ} \mathrm{C}$, after which an appropriate amount of water or aqueous $\mathrm{NaCl}$ solution was added to the dispersed xanthan to obtain the $1 \%$ solution. The resulting solutions were stirred gently until they became translucent.

\subsection{Measurement of Viscosity}

The wide shear-rate dependence of the solution viscosity was measured using a HAAKE MARS rotational viscometer equipped with a sensor having cone-plate geometry of the C35/1 type (with an angular $1^{\circ}$ and a gap $0.05 \mathrm{~mm}$ between a cone and a plate). The solutions were loaded on the sensor of the rotating cone-plate and held at a fixed temperature of 25 using a circulator. The measurement was performed over a course of shear rate changes, $0.005 \rightarrow 1000 \rightarrow 0.005 \mathrm{~s}^{-1}$. Data points in the shear rate region greater than $0.01 \mathrm{~s}^{-1}$ were obtained by confirming the steady state of viscosities expressed by on-line sampling.

Because of the peculiar gel-like structure of the biological polymer solution and the noisy data produced from this gellike material, it is usually difficult to achieve entirely steady state flow, especially at low shear rates. The following viscosity measurement was performed on a quasi-steady flow state, probably caused by the fragile structure of the polymer solution under the applied shear stress.

\section{RESULTS AND DISCUSSION}

\subsection{Viscosity Behavior}

The shear-rate dependences of the viscosities for the samples are shown in Figs. 1 and 2, where the shear rate $\dot{\gamma}$ and viscosity $\eta$ are represented in logarithmic coordinates. The figures illustrate the viscosity behavior for water as well as the aqueous $\mathrm{NaCl}(1 \mathrm{M})$ solution. In the figures, the unfilled and filled marks (circles) indicate measurements for $1 \%$ solutions at increasing and decreasing shear rates, respectively.

For solutions with same concentration (weight-mass/ volume), the viscosities of the samples dissolved in aqueous $\mathrm{NaCl}$ solutions were considerably lower than those of the samples dissolved in water.

The unfilled circles in Fig. 1 (data for shear rates lower than $500 \mathrm{~s}^{-1}$ are shown) indicate viscosity measurements for the increase sweep in the shear rates, $0.005 \rightarrow 1000 \mathrm{~s}^{-1}$. The filled circles in the figure indicate viscosity measurements for the decrease sweep of shear rates, $1000 \rightarrow 0.005 \mathrm{~s}^{-1}$.

The shear thickening phenomenon for viscosity at the initial stage of the measurement for increasing shear rates is expressed in Fig.1 for a xanthan solution, which was indicated by the arrow; such a phenomenon has also been demonstrated by Nishinari et al. for a xanthan solution ${ }^{11)}$ and by Capron et al. for the fermentation broth of xanthan ${ }^{22)}$.

Above a shear rate of $0.05 \mathrm{~s}^{-1}$ measured under steadyflow conditions, the viscosities were nearly the same for both increasing and decreasing shear rates. For the solution of xanthan dissolved in aqueous $\mathrm{NaCl}(1 \mathrm{M})$, the shear thickening phenomenon was not observed, and the discrepancy in the viscosity behavior was small as in the case of xanthan dissolved in water (see the unfilled and filled symbols in Fig. 2). Furthermore, because the concentration of xanthan was the same in both solutions, the viscosities in the highshear-rate region larger than $500 \mathrm{~s}^{-1}$ (data are not shown for this shear rate region in Figs. 1 and 2), in which the extent of entanglement between the polymers might be low, were nearly equal. In the low-shear-rate region, as mentioned above, the viscosities for xanthan dissolved in aqueous $\mathrm{NaCl}$ 
were considerably lower than those for xanthan dissolved in water.

The viscosity of most polymer solutions exhibits a shear thinning behavior, and is generally brought to a single nondimensional form as a function of shear rate with a set of values of viscosity and shear rate ${ }^{26)}$. This property suggests that the viscosity behaviors for various polymer solutions have the same underlying mechanism and show a generality. Viscosity, however, is expressed as the ratio of shear stress to shear rate on a flow plane under shear flow. From the shear rate dependences of viscosity for various polymer solutions,

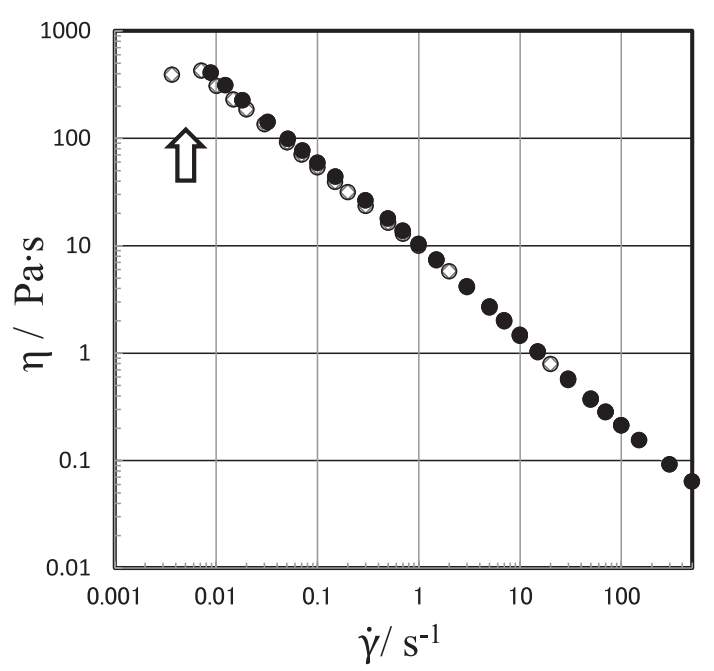

Fig. 1. Dependence of viscosity on shear rate for $1 \%$ xanthan solution dissolved in water (circular symbols).

Unfilled and filled symbols represent measurements with increasing and decreasing shear rates, respectively (as is also the case in Fig. 2).

The arrow in the figure indicates the portion of the shear thickening phenomenon, which is an increase of viscosity with increasing shear rate.

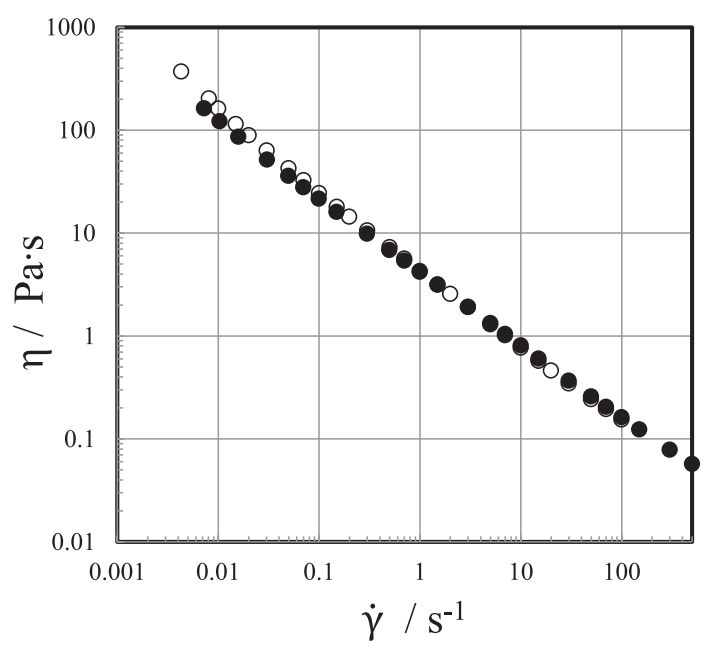

Fig. 2. Dependence of viscosity on shear rate for $1 \%$ xanthan solution dissolved in aqueous $\mathrm{NaCl}(1 \mathrm{M})$ solution (circular symbols). we can compare the value of the forces between dissolved polymers, which relates to the shear stress ${ }^{27}$. The following explanation is given on the basis of the fact that the viscosity behavior obeys the abovementioned interpretation.

\subsection{Correlation between Viscosity Behavior and Solution Structure}

The viscosity of a polymer solution is defined as the ratio of shear stress to shear rate. The force of the stress correlates with a contacting force between polymers in a shear flow. The shear stress appearing in a flow plane also correlates with forces traveling from every part of the fluid as a transmitting medium. Thus, in a polymer solution, the dependence of the viscosity on the shear rate is closely related to the fluid structure, i.e., whether polymer chains are in the single chain state or some chains are coagulated (associated) by molecular forces such as hydrogen bonds under the shear flow. The molecular weight of xanthan, which was undetermined in the present samples, is usually represented as an average value for dispersed polymers.

The shear stress produced by an applied shear rate affects the dissolved xanthan polymers. Therefore, the viscosity variation in the low-shear-rate range is probably caused by a change in the structure of the solution. The discrepancy between the viscosity data measured at increasing and decreasing shear rates may be due to imperfect sample preparation. However, a viscosity behavior similar to that found in this study has been shown for solutions of xanthan $^{11,22)}$, Aureobasidium pullulans cultures ${ }^{24)}$, etc. Thus, it is thought that this discrepancy is caused by a structure change inherent in these biological polymer solutions.

Dispersed polymers that are subjected to shear flow during viscosity measurements form lumps (a coagulated state) or dispersions (a single chain state) depending on whether the sites of contact between the polymers affected by the shear stress are fixed or separable. This interaction between the polymers is due to the forces that act between molecules, such as van der Waals forces, hydrogen bonding, and ionic interactions. It is assumed that a fixed connection is caused by an enforced interaction occurring in a certain molecular conformation under conditions of polymer entanglement in a low-stress environment. Although obtaining the molecular weights and concentrations of dissolved polymers is not an explicit aim of this study, these factors are considered with respect to the extent of polymer entanglement, which affects the viscosity level.

In Fig. 3, the parts surrounded by the large circles illustrate a structural change in a polymer solution loaded on a sensor 
disk, as a model of the xanthan solution. The curved lines randomly distributed in the circles are a simple representation of xanthan polymers with a double-helical structure. The structure of the solution including the xanthan double-helical structure was unchanged throughout the measurement process, because the temperature of the sensor disk was kept at $25^{\circ} \mathrm{C}$ and the shear stress acting on the polymer was not very high.

The abovementioned phenomenon is due to the fact that there is no conspicuous separation of the viscosities with increasing and decreasing shear rates over the wide shear rate range measured under steady shear rate flow $(0.01<\dot{\gamma}<$ $1000 \mathrm{~s}^{-1}$ ), for the $1 \%$ aqueous xanthan solution, as shown in Fig. 1 (presented for shear rates lessor than $500 \mathrm{~s}^{-1}$ ). The dependencies caused by increasing and decreasing shear rates should be expressed by different paths, provided that the double-helical structure of xanthan is affected by the applied shear stress.

The arrows in Fig. 3 indicate the measurement processes with increasing (A) and decreasing (B) shear rates. The small filled and unfilled circles represent sites of contact between the polymers that are fixed and separable, respectively, under an applied shear stress. In Figure 3, a-3 and a-4, b-1 and b-2, and so on differ only in their numbers of separable sites (i.e., the number for a-3 is greater than that for $\mathrm{a}-4$, and in case $\mathrm{B}$, the number is greater on the left-hand side of each figure, where the shear rate is lower). Therefore, this structural variation of the solution during measurement process is used in the following arguments to discuss the shear-rate dependences of the viscosity of the xanthan solutions shown in Figs. 1 and 2.

Clusters of polymers in contact with fixed sites form a gel (a-1) in a state of rest. As the shear rate increases, the shear

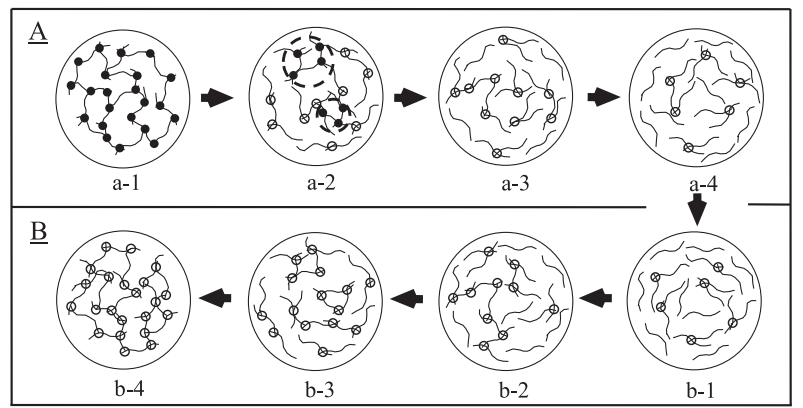

Fig. 3. Schematic illustration of a shear-dependent xanthan solution structure loaded on a circular-disc sensor.

The curved lines in the circles represent xanthan polymers, and small filled and unfilled circles represent fixed and separable contacting sites, respectively. The parts enclosed by dotted circles in a-2 represent lumps of xanthan polymers. The arrows indicate the measurement processes with increasing (A) and decreasing (B) shear rates. stress transforms the gel into polymer lumps (microgel). The parts enclosed by the dotted circle in a- 2 represent lumps or coagulated portions of the xanthan polymers. At a high shear-rate, these lumps are transformed into a dispersion of polymers with separable sites in the shear flow (a-3, a-4). When the shear rate decreases (B), the dispersive aspects of the polymers at high shear-rates $(b-1, b-2)$ are supposed to be similar to those of a-4 and a-3, with separable sites. This explains why the viscosities are nearly the same in this shearrate range (see Figs. 1 and 2). However, the viscosities vary at low shear-rates, indicating that the dispersive aspects of the polymers are based on different states. On the basis of this observation, it is assumed that the polymers retain a dispersive state with separable sites, even at low shear-rates (b-3, b-4). Hence, during the viscosity measurement, an external force in the form of the shearing flow is applied to individual xanthan macromolecules. This inhibits the formation of lumps of coagulated molecules (shown by the filled circles in Fig. 3). Because of the durability of the structural variations induced by the applied shear stress, a discrepancy arises between the viscosity data measured at increasing and decreasing shear rates, even when the experiment is repeated using the same sample after a certain sufficient resting time. At a high shear rate, and for a short resting time, the polymers are in the single chain state.

The viscosity behaviors of polymer solutions measured at increasing shear rates, as shown by the corresponding curves in the figures, are explained as follows. The viscosity of the polymer solution is mainly attributed to entanglement of the chains of dispersed polymers under shear flow. In general, solutions consisting of monodisperse polymers exhibit decreasing viscosity with increasing shear rates (shown by filled symbols in Fig. 1 for decreasing shear rates). The shear thickening phenomenon observed in the present and previous experiments $^{11,22)}$ for xanthan is accompanied by structural variations in the microgel under the applied shear stress ${ }^{29)}$.

With increasing shear rates, and because of shear stress under shear flow, the xanthan polymer starts aggregating into small lumps and macromolecures. Although this increases the polymer's viscosity, the viscosity of the polymer solution that now consists mostly of polymer lumps and has fewer polymer entanglements is low. On the other hand, monodisperse polymers, which cause the solution viscosity, increase with increasing shear rate due to the disruption of lumps. The increasing and decreasing viscosity with increasing shear rate indicated by the reported data in the low-shear-rate region can be attributed to a combination of the dynamical phenomena discussed above. 
Furthermore, as observed in the case of various biological polymer solutions ${ }^{11,24)}$, the solution viscosity measured at increasing shear rates exceeds the viscosity measured at decreasing shear rates. The following consideration will be useful in elucidating this unique trend in the viscosity.

The viscosity of a polymer solution is also correlated with the shear stress acting on the flow plane under shearing flow. This shear stress depends on the force exerted on the flow plane by every part of the solution ${ }^{27}$. In this case, the dissolved polymers and lumps (see a-2 in Fig. 3) may act as mediators of force transmittance. Under the experimental conditions, as the aforesaid force transmission process is applied, the viscosity measured at increasing shear rates for a given lump size may exceed that measured at decreasing shear rates.

\subsection{Effect of $\mathrm{NaCl}$ on Solution Viscosity}

While the concentration of xanthan polymer was equal to the weight-mass/volume of the pure water and aqueous $\mathrm{NaCl}$ solutions, in which the number of xanthan polymers per unit volume was equal, the viscosities of the $\mathrm{NaCl}$ solution were lower (for example, at the shear rate value $0.1 \mathrm{~s}^{-1}, 21.7 \mathrm{~Pa} \cdot \mathrm{s}$, instead of 54.0 Pa.s for the water). Because the viscosity of the solution results from the entanglement of the dissolved polymers, it is assumed that in these solutions the contacting force between the monodisperse xanthan polymers weakened.

The gradual variations in the viscosities in the low-shearrate range (Fig. 2) are also assumed to be caused by the weak interaction between the polymers, because polymer lumps are not easily formed under such conditions.

\section{CONCLUDING REMARKS}

A xanthan polymer has an extremely complicated structure and intriguing properties; this is a reflection of its origin from microorganisms. However, the shear-rate dependence of viscosity for xanthan solutions observed in this study is similar to that for Aureobasidium pullulans cultures mainly consisting of neutral polysaccharides, except that the culture viscosity is much smaller at a very low shear-rate. For instance, the viscosity is $2.5 \mathrm{~Pa} \cdot \mathrm{s}$ at the shear rate $0.1 \mathrm{~s}^{-1}$, for the pullulan concentration $0.58 \%(\mathrm{w} / \mathrm{v}){ }^{28)}$

The characteristics observed for the culture viscosity will result from the weak interactions between the dissolved polymers. On the other hand, it is surmised that in the xanthan solution, ionic interactions resulting from attractive and repulsive forces between the entangled anionic polysaccharides, which might be stronger than those between the neutral polysaccharides, are related to the viscosity behavior. Further, the rheological properties of xanthan-based solutions are affected by the environmental conditions, as the solution dissolves ions such as $\mathrm{K}^{+}$and $\mathrm{Ca}^{2+}$, in addition to $\mathrm{Na}^{+}$, and urea. ${ }^{1-5)}$

\section{ACKNOWLEDGEMENTS}

We wish to express thanks to Prof. Yasuhiro Matsuda of Shizuoka University for informative comments for xanthan solutions. We also wish to express thanks to EKO Instruments, Inc., for cooperation with viscosity measurement.

\section{REFERENCES}

1) Ross-Murphy SB, Morris VJ, Morris ER, Faraday Symp Chem Soc, 18, 115 (1983).

2) Smith IH, Symes KC, Lawson CJ, Morris ER, Int J Biol Macromol, 3, 129 (1981).

3) Richardson RK, Ross-Murphy SB, Int J Biol Macromol, 9, 257 (1987)

4) Callet F, Milas M, Rinaudo M, Int J Biol Macromol, 9, 291 (1987).

5) Shatwell KP, Sutherland IW, Ross-Murphy SB, Int J Biol Macromol, 12, 71 (1990).

6) Foss P, Stokke BT, Smidsrod O, Carbohydrate Polymers, 7, 421 (1987).

7) Kierulf C, Sutherland IW, Carbohydrate Polymers, 9, 185 (1988).

8) Lee HC, Brant DC, Macromolecules, 35, 2212 (2002).

9) Lee HC, Brant DC, Macromolecules, 35, 2223 (2002).

10) Nishinari K, Foods Food Ingredients J Japan, 212, 4 (2007).

11) Nishinari K, Takemasa M, Su L, Mizunuma H, Ogoshi H, Food Hydrocolloids, 25, 1737 (2011).

12) Jansson PE, Kenne L, Lindberg B, Carbohydr Res, 45, 275 (1975).

13) Holzwarth G, Biochemistry, 15, 4333 (1976).

14) Melton LD, Mindt L, Rees DA, Sanderson GR, Carbohydr Res, 46, 245 (1976).

15) Whitcomb PJ, Macosko CW, J Rheol, 22, 493 (1978).

16) Milas M, Rinaudo M, Carbohydr Res, 76, 189 (1979).

17) Liu W, Norisuye T, Int J Biol Macromol, 10, 44 (1988).

18) Milas M, Rinaudo M, Knipper M, Schuppiser JL, Macromolecules, 23, 2506 (1990).

19) Sato T, Kakihara T, Teramoto A, Polymer, 31, 824 (1990).

20) Oerter R, Kulicke WM, Rheol. Acta, 30, 140 (1991).

21) Inatomi S, Jinbo $Y$, Sato T, Teramoto A, Macromolecules, 25, 5013 (1992).

22) Capron I, Brigand G, Muller G, Int J Biol Macromol, 23, 215 (1998).

23) Matsuda Y, Biyajima Y, Sato T, Polymer Journal, 41, 526 (2009). 
24) Toda K, Gotoh Y, Asakura T, Yabe I, Furuse H, J Biosci Bioeng, 89, 258 (2000).

25) Li X, Fang Y, Al-Assaf S, Phillips GO, Nishinari K, Zhang H, Food Hydrocolloids, 23, 2394 (2009).

26) Morris ER, Carbohydrate Polymers, 13, 85 (1990).
27) Furuse H, Toda K, J Biosci Bioeng, 87, 218 (1999).

28) Furuse H, Yabe I, Asakura T, Miyawaki O, Toda K, J Biosci Bioeng, 95, 544 (2003).

29) Fang Y, Takahashi R, Nishinari K, Biopolymers, 74, 302 (2004). 